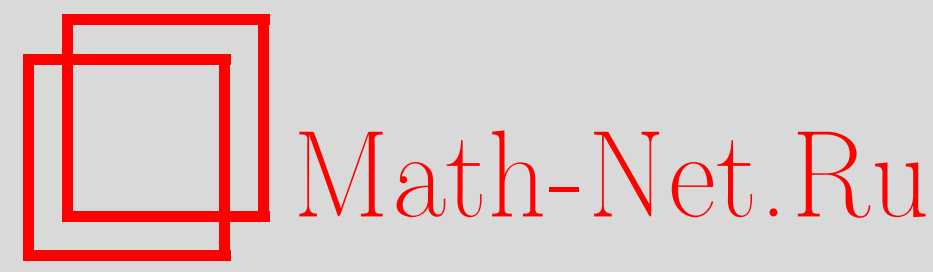

К. Ю. Осипенко, О точных значениях $n$-поперечников на классах, задаваемых операторами, не увеличивающими осцилляции, Матем. сб., 1997, том 188, номер 9, 113-126

DOI: https://doi.org/10.4213/sm259

Использование Общероссийского математического портала Math-Net.Ru подразумевает, что вы прочитали и согласны с пользовательским соглашением

http://www . mathnet.ru/rus/agreement

Параметры загрузки:

IP: 18.208 .226 .222

26 апреля 2023 г., 14:46:52 
УДК 517

\author{
К. Ю. Осипенко
}

\title{
О точных значениях $n$-поперечников на классах, задаваемых операторами, не увеличивающими осцилляции
}

\begin{abstract}
В работе предложен единый подход к задачам вычисления точных значений $n$-поперечников в равномерной метрике для классов периодических функций, задаваемых операторами (не обязательно линейньми), обладающими определенными осцилляционньми свойствами. Этот подход позволяет получать точные результаты об $n$-поперечниках как для классов функций, представимых в виде свертки с ядром, не увеличивающим осцилляции, так и для некоторых классов аналитических функций, которые не представляются в виде такой свертки.

Библиографйя: 11 названий.
\end{abstract}

\section{1. Введение}

Многие экстремальные задачи теории приближений на классах гладких периодических функций удается решить с помошью исследования осцилляционных свойств функций из этих классов. Подход, основанный на таких исследованиях, привел к изучению классов функций, представимых в виде свертки с ядрами, не повьшаюшими осцилляции. Для таких классов были получены довольно общие результаты, касаюшиеся оптимального восстановления, оптимальных квадратурных формул, $n$-поперечников, неравенств Колмогорова и ряд других (см. [1]-[3]).

Однако некоторые классы аналитических функций не удается представить в виде свертки с ядрами, не увеличивающими осцилляции. Тем не менее, на этих классах имеются результаты, весьма близкие к гладкому случаю (см. [4]-[6]). В данной работе для задачи вычисления точных значений $n$-поперечников предлагается единый подход, обслуживающий как гладкий, так и аналитический случаи, основанный на введении специального класса операторов (вообще говоря, нелинейных), обладающих свойством неувеличения осцилляции.

\section{2. Основные определения}

Напомним определения некоторых из $n$-поперечников. Пусть $A$ - подмножество линейного нормированного пространства $X$. Колмогоровским $n$-поперечником называется величина

$$
d_{n}(A, X):=\inf _{X_{n}} \sup _{x \in A} \inf _{y \in X_{n}}\|x-y\|,
$$

Работа выполнена при финансовой поддержке Российского фонда фундаментальных исследований (грант № 96-01-00325).

(C) К. Ю. ОСипенко 1997 
где $X_{n}$ - произвольные $n$-мерные подпространства $X$.

Линейным $n$-поперечником называется величина

$$
\lambda_{n}(A, X):=\inf _{Y} \inf _{P_{n}} \sup _{x \in A}\left\|x-P_{n} x\right\|
$$

где $Y$ - всевозможные линейные нормированные пространства, содержашие $A$, а $P_{n}$ - линейные ограниченные операторы, отображающие $Y$ в $X$, ранг которых не превосходит $n$.

Гельфандовский $n$-поперечник определяется следуюшим образом:

$$
d^{n}(A, X):=\inf _{Y} \inf _{Y^{n}} \sup _{x \in A \cap Y^{n}}\|x\|
$$

где $Y$ имеет тот же смысл, что и в определении линейного $n$-поперечника, а $Y^{n}$ - всевозможные подпространства $Y$ коразмерности $n$ (здесь предполагается, что $0 \in A)$.

Информационным $n$-поперечником множества $A$ назовем величину

$$
i_{n}(A, X):=\inf _{\substack{Y \supset A \\ l_{1}, \ldots, l_{n} \in Y^{*}}} \inf _{\mathscr{L}: \mathbb{R}^{n} \rightarrow X} \sup _{x \in A}\left\|x-\mathscr{L}\left(l_{1} x, \ldots, l_{n} x\right)\right\|_{X}
$$

где по-прежнему $Y$ - всевозможные линейные нормированные пространства, содержащие $A$. Линейные функционалы, на которых достигается нижняя гранњ в (1), будем называть оптимальными функционалами для соответствующего информационного поперечника.

Лемма 1. Пусть $A$ - иентрально-симметричное множество, содержащее нуль. Тогда

$$
d^{n}(A, X) \leqslant i_{n}(A, X) \leqslant \lambda_{n}(A, X)
$$

ДокАЗАТЕЛЬСтво. Неравенство

$$
i_{n}(A, X) \leqslant \lambda_{n}(A, X)
$$

непосредственно следует из определений информационного и линейного поперечников. Докажем оценку снизу. Пусть $Y \supset A$ и $l_{1}, \ldots, l_{n} \in Y^{*}$. Для любого $\varepsilon>0$ найдется $x_{\varepsilon} \in A$, для которого $l_{1} x_{\varepsilon}=\cdots=l_{n} x_{\varepsilon}=0$ и

$$
\sup _{\substack{x \in A \\ l_{1} x=\cdots=l_{n} x=0}}\|x\|_{X} \leqslant\left\|x_{\varepsilon}\right\|_{X}+\varepsilon
$$

Для всех $\mathscr{L}$ имеем

$$
\left\|x_{\varepsilon}-\mathscr{L}(0, \ldots, 0)\right\|_{X}+\left\|-x_{\varepsilon}-\mathscr{L}(0, \ldots, 0)\right\|_{X} \geqslant 2\left\|x_{\varepsilon}\right\|_{X}
$$


Поэтому

$$
\sup _{x \in A}\left\|x-\mathscr{L}\left(l_{1} x, \ldots, l_{n} x\right)\right\|_{X} \geqslant\left\|x_{\varepsilon}\right\|_{X} \geqslant \sup _{\substack{x \in A \\ l_{1} x=\cdots=l_{n} x=0}}\|x\|_{X}-\varepsilon \geqslant d^{n}(A, X)-\varepsilon
$$

Отсюда в силу произвольности $l_{1}, \ldots, l_{n}, \mathscr{L}$ и $\varepsilon>0$ получаем

$$
i_{n}(A, X) \geqslant d^{n}(A, X)
$$

Через $L_{p}, 1 \leqslant p \leqslant \infty$, обозначим пространство вещественных $2 \pi$-периодических функций, для которых

$$
\begin{aligned}
\|x\|_{p} & :=\left(\int_{\mathbb{T}}|x(t)|^{p} d t\right)^{1 / p}<\infty, \quad 1 \leqslant p<\infty \\
\|x\|_{\infty} & :=\operatorname{vraisup}|x(t)|<\infty, \quad p=\infty
\end{aligned}
$$

Положим

$$
B L_{\infty}:=\left\{x \in L_{\infty} \mid\|x\|_{\infty} \leqslant 1\right\}
$$

Обозначим через $\mathscr{F}$ множество операторов $F: B L_{\infty} \rightarrow L_{\infty}$ (вообще говоря, нелинейных), для которых

1) при всех $x \in B L_{\infty} F(-x)=-F x$;

2) при всех $\alpha \in \mathbb{T} P_{\alpha} \circ F=F \circ P_{\alpha}$, где $\left(P_{\alpha} x\right)(\cdot)=x(\cdot+\alpha)$;

3) $F$ непрерьвен как оператор, действующий из подмножества $B L_{\infty}$ пространства $L_{1}$ в $L_{1}$ (т. е. $\left\|F x_{m}-F x\right\|_{1} \rightarrow 0$ при $\left.\left\|x_{m}-x\right\|_{1} \rightarrow 0\right)$.

Для конечного, симметричного относительно нуля множества $M \subset \mathbb{Z}$ обозначим через $\mathscr{T}_{M}$ множество вещественных тригонометрических полиномов из $\operatorname{span}\left\{e^{i k t}\right\}_{k \in M}, \mathscr{T}_{\varnothing}:=\{0\}$. Пусть $G \in \mathscr{F}$, а $M_{0}$ и $M$ - конечные, симметричные относительно нуля множества целых чисел. Через $C V D\left(M_{0}, M, G\right)$ будем обозначать множество операторов $F \in \mathscr{F}$, для которых выполнены следующие условия:

1) если $x_{1}, x_{2}$ - различные функции из $B L_{\infty}$ такие, что $G x_{1} \perp \mathscr{T}_{M}, G x_{2} \perp \mathscr{T}_{M}$, a $p \in \mathscr{T}_{M_{0}}$, то

$$
S\left(p+F x_{1}-F x_{2}\right) \leqslant S\left(x_{1}-x_{2}\right)
$$

где $S(f)$ - число перемен знака $2 \pi$-периодической функции $f$ на периоде (относительно определения $S(f)$ для $f \in L_{\infty}$ см. [1, с. 41]);

2) при всех $0<\rho<1$ и всех $x \in B L_{\infty}$ таких, что $\|x\|_{\infty} \leqslant 1$ и $G x \perp \mathscr{T}_{M}$, существует функция $x_{\rho} \in B L_{\infty}$ такая, что $\left\|x_{\rho}\right\|_{\infty}<1, G x_{\rho} \perp \mathscr{T}_{M}$ и $\rho F x=F x_{\rho}$.

3) множество $\left\{F x \mid x \in B L_{\infty}, G x \perp \mathscr{T}_{M}\right\}$ выпукло;

4) если $x \in B L_{\infty}$ и $G x \perp \mathscr{T}_{M}$, то $F x \perp \mathscr{T}_{M}$. 
Последнее условие выполнено, например, если $G$ - тождественный оператор, а $F$ - оператор свертки, или если $G=F$. Эти два случая являются основными в рассматриваемых ниже примерах.

Для функции $f \in C(\mathbb{T})$ через dist $f$ обозначим максимальную длину подинтервала $\mathbb{T}$, на котором функция $f$ не имеет нулей. Положим

$$
(f * g)(t):=\frac{1}{2 \pi} \int_{\mathbb{T}} f(t-s) g(s) d s .
$$

Обозначим через $\mathscr{K}(M, \delta)$ класс ядер $\Omega \in L_{1}$, для которых при всех $x \in L_{\infty}$ и $p \in \mathscr{T}_{M}$ таких, что $x \perp \mathscr{T}_{M}, x \not \equiv 0$, a $\operatorname{dist}(p+\Omega * x)<\delta$, вьполнено неравенство

$$
S(p+\Omega * x) \leqslant S(x),
$$

причем, если $\Omega * x \in C^{2}(\mathbb{T})$, то

$$
Z_{2}(p+\Omega * x) \leqslant S(x),
$$

где $Z_{2}$ - число нулей функции, когда кратные нули считаются дважды, а интервалы, на которых функция тождественно равна нулю, отбрасываются. Будем также предполагать, что $c_{j}(\Omega) \neq 0, j \notin M$, где

$$
c_{j}(f):=\frac{1}{2 \pi} \int_{\mathbb{T}} f(t) e^{-i j t} d t, \quad j=0, \pm 1, \ldots .
$$

Предположим, что

$$
\Omega_{j} \in \mathscr{K}\left(M_{j}, \delta_{j}\right), \quad j=1, \ldots, k, \quad F \in C V D\left(M_{0}, M, G\right), \quad M=\bigcup_{j=0}^{k} M_{j} .
$$

Нас будут интересовать точные значения $n$-поперечников для классов

$$
\mathscr{F}_{\infty}:=\mathscr{F}_{\infty}(\Phi, M):=\left\{p+\Phi x \mid p \in \mathscr{T}_{M}, x \in B L_{\infty}, G x \perp \mathscr{T}_{M}\right\},
$$

где $\Phi x=\Omega_{k} * \cdots * \Omega_{1} * F x$.

\section{3. Точные значения $n$-поперечников на классах $\mathscr{F}_{\infty}$}

Пусть $n \in \mathbb{N}$ и

$$
\Theta_{n}:=\left\{\theta \mid \theta=\left(\theta_{1}, \ldots, \theta_{n}\right), 0 \leqslant \theta_{1} \leqslant \cdots \leqslant \theta_{n}<2 \pi\right\} .
$$

Для $\eta \in \Theta_{2 n}$ положим

$$
h_{\eta}(t):=(-1)^{j}, \quad t \in\left[\eta_{j-1}, \eta_{j}\right), \quad j=1, \ldots, 2 n+1,
$$

где $\eta_{0}:=0, \eta_{2 n+1}:=2 \pi$. Через $h_{n}(t)$ обозначим функцию $h_{\eta}(t)$, когда $\eta_{j}=$ $(j-1) \pi / n, j=1, \ldots, 2 n$.

Будем предполагать вьполненными условия (3). Положим

$$
\delta:=\min _{1 \leqslant j \leqslant k} \delta_{j}, \quad m:= \begin{cases}\sup \{j \mid j \in M\}, & M \neq \varnothing, \\ -1, & M=\varnothing .\end{cases}
$$

Лемма 2. Для всех $n>\max \{m, 2 \pi / \delta\}$ имеет место равенство

$$
\inf _{\substack{p \in \mathscr{T}_{M} \\ 2 n, G h_{\eta} \perp \mathscr{T}_{M}}}\left\|p+\Phi h_{\eta}\right\|_{\infty}=\left\|\Phi h_{n}\right\|_{\infty}
$$


ДоКАЗАТЕЛЬСТво. Положим

$$
G_{\sigma}(t)=\frac{\sqrt{2 \pi}}{\sigma} \sum_{j \in \mathbb{Z}} \exp \left(-\frac{(t-2 \pi j)^{2}}{2 \sigma^{2}}\right)=1+2 \sum_{j=1}^{\infty} e^{-j^{2} \sigma^{2} / 2} \cos j t, \quad \sigma>0 .
$$

Хорошо известны следующие свойства ядра $G_{\sigma}$ (см. [7]): при всех $f \in L_{\infty} G_{\sigma} * f$ - аналитические функции и, кроме того,

$$
\lim _{\sigma \rightarrow 0}\left\|G_{\sigma} * f\right\|_{\infty}=\|f\|_{\infty}, \quad Z\left(G_{\sigma} * f\right) \leqslant S(f)
$$

где $Z(g)$ - число нулей функции $g$ с учетом кратности. Предположим, что существуют $p \in \mathscr{T}_{M}$ и $\eta \in \Theta_{2 n}$, для которых $G h_{\eta} \perp \mathscr{T}_{M}$ и

$$
\left\|p+\Phi h_{\eta}\right\|_{\infty}<\left\|\Phi h_{n}\right\|_{\infty}
$$

Тогда при достаточно малых $\sigma$

$$
\left\|p_{\sigma}+\Phi_{\sigma} h_{\eta}\right\|_{\infty}<\left\|\Phi_{\sigma} h_{n}\right\|_{\infty}
$$

где $p_{\sigma}=G_{\sigma} * p \in \mathscr{T}_{M}, \Phi_{\sigma} x=G_{\sigma} * \Phi x$. В силу свойств свертки и оператора $F$ имеем

$$
P_{\pi / n}\left(\Phi_{\sigma} h_{n}\right)=\Phi_{\sigma}\left(P_{\pi / n} h_{n}\right)=\Phi_{\sigma}\left(-h_{n}\right)=-\Phi_{\sigma} h_{n} .
$$

Отсюда следует существование точек $0 \leqslant t_{1}<\cdots<t_{2 n}<2 \pi$ таких, что

$$
\left(\Phi_{\sigma} h_{n}\right)\left(t_{j}\right)=\varepsilon(-1)^{j}\left\|\Phi_{\sigma} h_{n}\right\|_{\infty}, \quad j=1, \ldots, 2 n,
$$

где $\varepsilon=1$ или -1 . Тем самьм для любого $\alpha \in \mathbb{T}$

$$
2 n \leqslant S\left(P_{\alpha}\left(\Phi_{\sigma} h_{n}\right)-p_{\sigma}-\Phi_{\sigma} h_{\eta}\right)=S\left(\Phi_{\sigma}\left(P_{\alpha} h_{n}\right)-p_{\sigma}-\Phi_{\sigma} h_{\eta}\right)
$$

Если при каком-либо $\alpha h_{\eta}=P_{\alpha} h_{n}$, то

$$
2 n \leqslant S\left(P_{\alpha}\left(\Phi_{\sigma} h_{n}\right)-p_{\sigma}-\Phi_{\sigma} h_{\eta}\right)=S(p) \leqslant 2 m<2 n
$$

Тем самым для всех $\alpha P_{\alpha} h_{n} \neq h_{\eta}$.

Из того, что $c_{s}\left(\Omega_{j}\right) \neq 0, s \notin M_{j}$, следует существование тригонометрических полиномов $p_{j} \in \mathscr{T}_{M_{j}}, j=0, \ldots, k$, для которых

$$
p_{\sigma}+\Phi_{\sigma} h_{\eta}=p_{k}+\Omega_{k} *\left(p_{k-1}+\cdots+\Omega_{1} * G_{\sigma} *\left(p_{0}+F h_{\eta}\right) \cdots\right) .
$$

Положим

$$
\begin{gathered}
f_{j}:=p_{j}+\Omega_{j} *\left(p_{j-1}+\cdots+\Omega_{1} * G_{\sigma} *\left(p_{0}+F h_{\eta}\right) \cdots\right), \quad j=1, \ldots, k, \\
g_{j}:=\Omega_{j} * \cdots * \Omega_{1} * G_{\sigma} * F h_{n}, \quad j=1, \ldots, k, \\
f_{0}:=G_{\sigma} *\left(p_{0}+F h_{\eta}\right), \quad g_{0}:=G_{\sigma} * F h_{n}, \\
\mu_{j}:=\frac{\left\|f_{j}\right\|_{\infty}}{\left\|g_{j}\right\|_{\infty}}, \quad j=0, \ldots, k-1, \quad \mu:=\max _{0 \leqslant j \leqslant k-1} \mu_{j} .
\end{gathered}
$$


Докажем, что $\mu_{0} \geqslant 1$. Предположим противное. Тогда аналогично предыдушим рассуждениям (см. (4)-(6)) можно показать, что при всех $\alpha \in \mathbb{T}$

$$
S\left(P_{\alpha} g_{0}-f_{0}\right) \geqslant 2 n \text {. }
$$

Выбрав $\alpha=-\eta_{1}$, имеем

$$
\begin{aligned}
2 n \leqslant S\left(G_{\sigma} * F\left(P_{\alpha} h_{n}\right)-G_{\sigma} *\left(p_{0}+F h_{\eta}\right)\right) & \leqslant S\left(F\left(P_{\alpha} h_{n}\right)-p_{0}-F h_{\eta}\right) \\
& \leqslant S\left(h_{n}(\cdot+\alpha)-h_{\eta}(\cdot)\right) \leqslant 2(n-1) .
\end{aligned}
$$

Тем самым доказано, что $\mu \geqslant 1$. Пусть $\mu=\mu_{s}, 0 \leqslant s \leqslant k-1$. Выберем $\alpha \in \mathbb{T}$ так, чтобы разность

$$
P_{\alpha} g_{s}-\mu_{s}^{-1} f_{s}
$$

имела кратный нуль. Аналогично равенствам (4) получаем

$$
P_{\pi / n} g_{j}=-g_{j}
$$

Отсюда вытекает, что

$$
\operatorname{dist}\left(P_{\alpha} g_{j}-\mu_{s}^{-1} f_{j}\right) \leqslant \frac{2 \pi}{n}<\delta .
$$

Следовательно, в силу свойств ядер $\Omega_{j}$ при $s>0$ имеем

$$
\begin{aligned}
2 n & \leqslant S\left(P_{\alpha} g_{k}-\mu_{s}^{-1} f_{k}\right) \leqslant \cdots \leqslant S\left(P_{\alpha} g_{s}-\mu_{s}^{-1} f_{s}\right)<Z_{2}\left(P_{\alpha} g_{s}-\mu_{s}^{-1} f_{s}\right) \\
& \leqslant S\left(P_{\alpha} g_{s-1}-\mu_{s}^{-1} f_{s-1}\right) \leqslant \cdots \leqslant S\left(P_{\alpha} g_{0}-\mu_{s}^{-1} f_{0}\right) \\
& \leqslant S\left(F\left(P_{\alpha} h_{n}\right)-\mu_{s}^{-1}\left(p_{0}+F h_{\eta}\right)\right) .
\end{aligned}
$$

Если $\mu_{s}>1$, то по свойству 2) операторов из множества $C V D\left(M_{0}, M, G\right)$ найдется функция $h^{*} \in B L_{\infty}$ такая, что $\left\|h^{*}\right\|_{\infty}<1, G h^{*} \perp \mathscr{T}_{M}$ и $\mu_{s}^{-1} F h_{\eta}=F h^{*}$. При $\mu_{s}=1$ положим $h^{*}=h_{\eta}$. Таким образом,

$$
S\left(F\left(P_{\alpha} h_{n}\right)-\mu_{s}^{-1}\left(p_{0}+F h_{\eta}\right)\right) \leqslant S\left(h_{n}(\cdot+\alpha)-h^{*}(\cdot)\right) \leqslant 2 n,
$$

что противоречит (8). Если $s=0$, то неравенства (8) заменяются на следующие

$$
\begin{gathered}
2 n \leqslant S\left(P_{\alpha} g_{k}-\mu_{0}^{-1} f_{k}\right) \leqslant \cdots \leqslant S\left(P_{\alpha} g_{0}-\mu_{0}^{-1} f_{0}\right)<Z_{2}\left(P_{\alpha} g_{0}-\mu_{0}^{-1} f_{0}\right) \\
\leqslant S\left(F\left(P_{\alpha} h_{n}\right)-\mu_{s}^{-1}\left(p_{0}+F h_{\eta}\right)\right) .
\end{gathered}
$$

Тем самым доказано, что

$$
\left\|p+\Phi h_{\eta}\right\|_{\infty} \geqslant\left\|\Phi h_{n}\right\|_{\infty}
$$

Из равенства

$$
P_{\pi / n} G h_{n}=-G h_{n}
$$

вытекает, что $G h_{n}-2 \pi / n$-периодическая функция. Поэтому $G h_{n} \perp \mathscr{T}_{M}$.

Положим $\mathscr{T}_{n}:=\mathscr{T}_{[-n, n] \cap \mathbb{Z},}$

$$
\begin{aligned}
a_{j}(f) & :=\frac{1}{\pi} \int_{\mathbb{T}} f(t) \cos j t d t, \quad j=0,1, \ldots, \\
b_{j}(f) & :=\frac{1}{\pi} \int_{\mathbb{T}} f(t) \sin j t d t, \quad j=1,2, \ldots, \\
I_{2 n-1}(f) & :=\left(a_{0}(f), a_{1}(f), b_{1}(f), \ldots, a_{n-1}(f), b_{n-1}(f)\right) .
\end{aligned}
$$

В дальнейшем будем предполагать, что при $k=0 F\left(B L_{\infty}\right) \subset C(\mathbb{T})$ и, кроме того, оператор $F$ непрерывен как оператор, действующий из подмножества $B L_{\infty}$ пространства $L_{1}$ в $C(\mathbb{T})$ (т.е. $\left\|F x_{m}-F x\right\|_{\infty} \rightarrow 0$ при $\left.\left\|x_{m}-x\right\|_{1} \rightarrow 0\right)$. 
Лемма 3. При всех $n>\max \{m, 2 \pi / \delta\}$ существует линейный оператор $\mathscr{L}: \mathbb{T}^{2 n-1} \rightarrow \mathscr{T}_{n-1}$, для которого

$$
\sup _{f \in \mathscr{F}_{\infty}}\left\|f-\mathscr{L}\left(I_{2 n-1}(f)\right)\right\|_{\infty}=\left\|\Phi h_{n}\right\|_{\infty}
$$

ДокаЗАТЕЛьство. Докажем сначала, что

$$
\sup _{\substack{f \in \mathscr{F}_{\infty} \\ I_{2 n-1}(f)=0}}\|f\|_{\infty}=\left\|\Phi h_{n}\right\|_{\infty}
$$

Предположим, что найдется функция $f \in \mathscr{F}_{\infty}$, для которой $I_{2 n-1}(f)=0$ и $\|f\|_{\infty}>\left\|\Phi h_{n}\right\|_{\infty}$. Пусть $f=\Phi x, x \in B L_{\infty}$. Тогда при достаточно малых $\sigma$ $\left\|\Phi_{\sigma} x\right\|_{\infty}>\left\|\Phi_{\sigma} h_{n}\right\|_{\infty}$. Оставив за $g_{j}$ те же обозначения, что и в лемме 2 , положим

$$
\begin{gathered}
f_{j}:=\Omega_{j} * \cdots * \Omega_{1} * G_{\sigma} * F x, \quad j=1, \ldots, k, \quad f_{0}:=G_{\sigma} * F x \\
\nu_{j}:=\frac{\left\|f_{j}\right\|_{\infty}}{\left\|g_{j}\right\|_{\infty}}, \quad j=0, \ldots, k, \quad \nu:=\max _{0 \leqslant j \leqslant k} \nu_{j} .
\end{gathered}
$$

Пусть $\nu=\nu_{s}, 0 \leqslant s \leqslant k$. В силу наших предположений $\nu_{s}>1$. Выберем $\alpha \in \mathbb{T}$ так, чтобы разность

$$
P_{\alpha} g_{s}-\nu_{s}^{-1} f_{s}
$$

имела кратный нуль. Из равенств $(7)$ вытекает, что функции $g_{j}$ имеют период $2 \pi / n$. Следовательно, $I_{2 n-1}\left(g_{j}\right)=0$. Тем самым

$$
I_{2 n-1}\left(P_{\alpha} g_{s}-\nu_{s}^{-1} f_{s}\right)=0 .
$$

Так как тригонометрическая система является чебышевской, то отсюда следует (см. $[1$, с. 41$])$, что

$$
S\left(P_{\alpha} g_{s}-\nu_{s}^{-1} f_{s}\right) \geqslant 2 n
$$

В силу того, что $\operatorname{dist}\left(P_{\alpha} g_{s}-\nu_{s}^{-1} f_{s}\right) \leqslant 2 \pi / n<\delta$, имеем

$$
\begin{aligned}
2 n & \leqslant S\left(P_{\alpha} g_{s}-\nu_{s}^{-1} f_{s}\right)<Z_{2}\left(P_{\alpha} g_{s}-\nu_{s}^{-1} f_{s}\right) \\
& \leqslant S\left(P_{\alpha} g_{s-1}-\nu_{s}^{-1} f_{s-1}\right) \leqslant \cdots \leqslant S\left(P_{\alpha} g_{0}-\nu_{s}^{-1} f_{0}\right) \\
& \leqslant S\left(F\left(P_{\alpha} h_{n}\right)-\nu_{s}^{-1} F x\right) \leqslant S\left(h_{n}(\cdot+\alpha)-x^{*}(\cdot)\right)=2 n,
\end{aligned}
$$

где $x^{*}$ определяется равенством $\nu_{s}^{-1} F x=F x^{*},\left\|x^{*}\right\|_{\infty}<1, G x^{*} \perp \mathscr{T}_{M}$. Полученное противоречие доказывает равенство (9).

Рассмотрим теперь задачу об оптимальном восстановлении значения $f(0)$ на классе $\mathscr{F} \infty$ по информации $I_{2 n-1}(f)$. Из общих результатов, касающихся задач 
восстановления, вытекает существование линейного оптимального метода восстановления, т.е. таких чисел $\alpha_{0}, \alpha_{1}, \beta_{1}, \ldots, \alpha_{n-1}, \beta_{n-1}$, что

$$
\begin{gathered}
\sup _{f \in \mathscr{F}_{\infty}}\left|f(0)-\alpha_{0} a_{0}(f)-\sum_{j=1}^{n-1}\left(\alpha_{j} a_{j}(f)+\beta_{j} b_{j}(f)\right)\right| \\
=\sup _{\substack{f \in \mathscr{F} \infty \\
I_{2 n-1}(f)=0}}\|f\|_{\infty}=\left\|\Phi h_{n}\right\|_{\infty} .
\end{gathered}
$$

Пусть $g$ - произвольная функция из $\mathscr{F}$. Для $t \in \mathbb{T}$ положим $f_{t}(\tau):=g(t+\tau)$. Так как $f_{t} \in \mathscr{F}_{\infty}, a_{0}\left(f_{t}\right)=a_{0}(g)$ и

$$
\begin{aligned}
& a_{j}\left(f_{t}\right)=a_{j}(g) \cos j t+b_{j}(g) \sin j t, \\
& b_{j}\left(f_{t}\right)=-a_{j}(g) \sin j t+b_{j}(g) \cos j t,
\end{aligned}
$$

то, положив

$$
\begin{aligned}
& \mathscr{L}\left(I_{2 n-1}(g)\right) \\
& :=\alpha_{0} a_{0}(g)+\sum_{j=1}^{n-1}\left(\left(\alpha_{j} \cos j t-\beta_{j} \sin j t\right) a_{j}(g)+\left(\alpha_{j} \sin j t+\beta_{j} \cos j t\right) b_{j}(g)\right),
\end{aligned}
$$

из (10) при $\tau=0$ будем иметь

$$
\left|g(t)-\mathscr{L}\left(I_{2 n-1}(g)\right)\right| \leqslant\left\|\Phi h_{n}\right\|_{\infty}
$$

В силу произвольности $t \in \mathbb{T}$ получаем

$$
\left\|g-\mathscr{L}\left(I_{2 n-1}(g)\right)\right\|_{\infty} \leqslant\left\|\Phi h_{n}\right\|_{\infty}
$$

Так как для $g=\Phi h_{n}$ последнее неравенство обрашается в равенство, то утверждение леммы доказано.

Теперь докажем основной результат работы.

ТЕОРема 4. При всех $n>\max \{m, 2 \pi / \delta\}$ имеют место равенства

$$
\begin{aligned}
d_{2 n}\left(\mathscr{F}_{\infty}, L_{\infty}\right) & =\lambda_{2 n}\left(\mathscr{F}_{\infty}, L_{\infty}\right)=d^{2 n}\left(\mathscr{F}_{\infty}, L_{\infty}\right)=i_{2 n}\left(\mathscr{F}_{\infty}, L_{\infty}\right) \\
& =d_{2 n-1}\left(\mathscr{F}_{\infty}, L_{\infty}\right)=\lambda_{2 n-1}\left(\mathscr{F}_{\infty}, L_{\infty}\right) \\
& =d^{2 n-1}\left(\mathscr{F}_{\infty}, L_{\infty}\right)=i_{2 n-1}\left(\mathscr{F}_{\infty}, L_{\infty}\right)=\left\|\Phi h_{n}\right\|_{\infty} .
\end{aligned}
$$

При этом коэффициенть Фурье $a_{0}(f), a_{1}(f), b_{1}(f), \ldots, a_{n-1}(f), b_{n-1}(f)$ являются оптимальными функционалами для величин $i_{2 n-1}$ u $i_{2 n}$. 
ДокАЗАТЕЛЬСтво. Докажем сначала оценку снизу для колмогоровского и гельфандовского $2 n$-поперечников. Положим

$$
\begin{gathered}
S^{2 n}:=\left\{\xi=\left(\xi_{1}, \ldots, \xi_{2 n+1}\right) \in \mathbb{R}^{2 n+1}\left|\sum_{s=1}^{2 n+1}\right| \xi_{s} \mid=2 \pi\right\} \\
\tau_{0}(\xi):=0, \quad \tau_{j}(\xi):=\sum_{s=1}^{j}\left|\xi_{s}\right|, \quad j=1, \ldots, 2 n+1 .
\end{gathered}
$$

Для $\xi \in S^{2 n}$ определим функции

$$
g_{\xi}(t):=\operatorname{sign} \xi_{j}, \quad \tau_{j-1}(\xi) \leqslant t<\tau_{j}(\xi), \quad j=1, \ldots, 2 n+1, \quad f_{\xi}:=\Phi g_{\xi} .
$$

Пусть $X_{2 n}$ - произвольное $2 n$-мерное подпространство $L_{q}, 1<q<\infty$. Предположим, что $X_{2 n}=\operatorname{span}\left\{f_{1}, \ldots, f_{2 n}\right\}$ и

$$
f_{\xi}^{*}:=\sum_{j=1}^{2 n} \alpha_{j}(\xi) f_{j}
$$

- элемент наилучшего приближения $f_{\xi}$ подпространством $X_{2 n}$. Положим

$$
E\left(\mathscr{F}_{\infty}, X_{2 n}\right):=\sup _{f \in \mathscr{F}_{\infty}} \inf _{g \in X_{2 n}}\|f-g\|_{q}
$$

Если $\mathscr{T}_{M} \not \subset X_{2 n}$, то $E\left(\mathscr{F}_{\infty}, X_{2 n}\right)=\infty$. Будем считать, что $\mathscr{T}_{M} \subset X_{2 n}$, $\operatorname{dim} \mathscr{T}_{M}=r$ и $\left\{f_{j}\right\}_{j=1}^{r}-$ базис $\mathscr{T}_{M}$. Положим $I_{M}(f):=\left\{a_{j}(f), b_{j}(f)\right\}_{j \in M \cap \mathbb{Z}_{+}}$. Рассмотрим отображение

$$
\alpha(\xi):=\left(I_{M}\left(G g_{\xi}\right), \alpha_{r+1}(\xi), \ldots, \alpha_{2 n}(\xi)\right) .
$$

Отображение $\alpha: S^{2 n} \rightarrow \mathbb{R}^{2 n}$ - непрерывное и нечетное. Поэтому по теореме Борсука сушествует $\xi^{*} \in S^{2 n}$, для которого $\alpha\left(\xi^{*}\right)=0$. Имеем

$$
\begin{aligned}
E\left(\mathscr{F}_{\infty}, X_{2 n}\right) \geqslant \inf _{g \in X_{2 n}}\left\|f_{\xi^{*}}-g\right\|_{q} & =\left\|\Phi g_{\xi^{*}}-\sum_{j=1}^{r} \alpha_{j}\left(\xi^{*}\right) f_{j}\right\|_{q} \\
& \geqslant \inf _{\substack{p \in \mathscr{T}_{M} \\
\eta \in \Theta_{2 n}, G h_{\eta} \perp \mathscr{T}_{M}}}\left\|p+\Phi h_{\eta}\right\|_{q} .
\end{aligned}
$$

Следовательно,

$$
d_{2 n}\left(\mathscr{F}_{\infty}, L_{q}\right) \geqslant \inf _{\substack{p \in \mathscr{T}_{M} \\ \eta \in \Theta_{2 n}, G h_{\eta} \perp \mathscr{T}_{M}}}\left\|p+\Phi h_{\eta}\right\|_{q}
$$


Переходя к пределу при $q \rightarrow \infty$ и пользуясь леммой 2 , будем иметь

$$
d_{2 n}\left(\mathscr{F}_{\infty}, L_{\infty}\right) \geqslant \inf \left\|p+\Phi h_{\eta}\right\|_{\infty}=\left\|\Phi h_{n}\right\|_{\infty} .
$$

Получим теперь оценку снизу для гельфандовских $2 n$-поперечников. Пусть $Y-$ некоторое линейное нормированное пространство, содержащее $F_{\infty}$, и

$$
X^{2 n}=\left\{f \in Y \mid\left\langle l_{j}, f\right\rangle=0, j=1, \ldots, 2 n, l_{j} \in Y^{*}\right\} .
$$

Рассмотрим отображение $J: \mathscr{T}_{M} \rightarrow \mathbb{R}^{2 n}$, задаваемое равенством

$$
J p=\left(l_{1} p, \ldots, l_{2 n} p\right) .
$$

Если $\operatorname{Ker} J \neq 0$, то

$$
\sup _{f \in \mathscr{F}_{\infty} \cap X^{2 n}}\|f\|_{\infty}=\infty .
$$

Если же Ker $J=0$, то среди функционалов $l_{1}, \ldots, l_{2 n}$ существуют $r$ линейно независимых на $\mathscr{T}_{M}$. Будем считать, что таковыми являются $l_{1}, \ldots, l_{r}$. Тогда остальные функционалы на $\mathscr{T}_{M}$ могут быть представлены в виде

$$
\left\langle l_{j}, p\right\rangle=\sum_{s=1}^{r} c_{j s}\left\langle l_{s}, p\right\rangle, \quad j=r+1, \ldots, 2 n .
$$

Положим

$$
L_{j}:=l_{j}-\sum_{s=1}^{r} c_{j s} l_{s}, \quad j=r+1, \ldots, 2 n
$$

и рассмотрим отображение

$$
\alpha(\xi):=\left(I_{M}\left(G f_{\xi}\right),\left\langle L_{r+1}, f_{\xi}\right\rangle, \ldots,\left\langle L_{2 n}, f_{\xi}\right\rangle\right) .
$$

По теореме Борсука сушествует $\xi^{*} \in S^{2 n}$, для которого $\alpha\left(\xi^{*}\right)=0$. Так как Ker $J=0$, то найдется тригонометрический полином $p^{*} \in \mathscr{T}_{M}$ такой, что

$$
\left\langle l_{s}, p^{*}\right\rangle=-\left\langle l_{s}, f_{\xi^{*}}\right\rangle, \quad s=1, \ldots, r .
$$

Для $r+1 \leqslant j \leqslant 2 n$ имеем

$$
\left\langle l_{j}, p^{*}+f_{\xi^{*}}\right\rangle=\left\langle L_{j}+\sum_{s=1}^{r} c_{j s} l_{s}, p^{*}+f_{\xi^{*}}\right\rangle=\sum_{s=1}^{r} c_{j s}\left\langle l_{s}, p^{*}+f_{\xi^{*}}\right\rangle=0 .
$$

Следовательно,

$$
p^{*}+f_{\xi^{*}} \in X^{2 n}
$$

Тем самым

$$
\sup _{f \in \mathscr{F}_{\infty} \cap X^{2 n}}\|f\|_{\infty} \geqslant\left\|p^{*}+f_{\xi^{*}}\right\|_{\infty} \geqslant \inf _{\substack{p \in \mathscr{T}_{M} \\ \eta \in \Theta_{2 n}, G h_{\eta} \perp \mathscr{T}_{M}}}\left\|p+\Phi h_{\eta}\right\|_{\infty}=\left\|\Phi h_{n}\right\|_{\infty} .
$$

Отсюда

$$
d^{2 n}\left(\mathscr{F}_{\infty}, L_{\infty}\right) \geqslant\left\|\Phi h_{n}\right\|_{\infty} .
$$

Из неравенств (2) и монотонности поперечников следует, что остается оценить сверху $\lambda_{2 n-1}\left(\mathscr{F}_{\infty}, L_{\infty}\right)$. Эта оценка вытекает из леммы 3. 


\section{4. Примеры классов $\mathscr{F}_{\infty}$}

Для $\Omega \in L_{1}$ положим

$$
W_{M}(\Omega):=\left\{p+\Omega * x \mid p \in \mathscr{T}_{M}, x \in B L_{\infty}, x \perp \mathscr{T}_{M}\right\}
$$

Назовем функцию $\Omega \in L_{1}$ ядром, не увеличивающим осцилляции, если при всех $x \in L_{\infty}$ таких, что $x \perp \mathscr{T}_{M}, x \not \equiv 0$, и при всех $p \in \mathscr{T}_{M}$ имеет место неравенство

$$
S(p+\Omega * x) \leqslant S(x) .
$$

Очевидно, что оператор $F x=\Omega * x$ принадлежит классу $C V D(M, M, \mathrm{Id})$ (Id - тождественный оператор). Следовательно,

$$
W_{M}(\Omega)=\mathscr{F}_{\infty}(\Phi, M),
$$

где $\Phi x=F x(k=0, G=\mathrm{Id})$.

При $M=\varnothing$ ядра, не увеличиваюшие осцилляции, называются функциями плотности, не увеличивающими осиилляции, или $C V D$-ядрами (cyclic variation diminishing kernels). Для классов функций, представимых в виде свертки с ядрами такого типа, теорема 4 доказана А. Пинкусом [1, с. 179].

Пусть $Q(D)$ - дифференциальный полином с постоянными вешественными коэффициентами

$$
Q(D)=D^{\operatorname{deg} Q}+\sum_{m=0}^{\operatorname{deg} Q-1} a_{m} D^{m}, \quad D=\frac{d}{d x} .
$$

Положим

$$
\Omega_{Q}(t):=\sum_{\substack{m \in \mathbb{Z} \\ Q(i m) \neq 0}} \frac{e^{i m t}}{Q(i m)}
$$

Пусть

$$
M=\{m \in \mathbb{Z} \mid Q(i m)=0\}
$$

Тогда

$$
W_{M}\left(\Omega_{Q}\right)=W_{\infty}^{Q}:=\left\{f \mid f^{(\operatorname{deg} Q-1)}-\text { абсолютно непрерывна, }\|Q(D) f\|_{\infty} \leqslant 1\right\} .
$$

Обозначим через $h(Q)$ максимум мнимой части нулей полинома $Q$. Из работ [8] и [3] вытекает, что при $h(Q) \leqslant 1 / 2$ ядро $\Omega_{Q}$ не увеличивает осцилляции (при этом $M=0$, если $Q(0)=0$, и $M=\varnothing$, если $Q(0) \neq 0)$. Таким образом, при $h(Q) \leqslant 1 / 2$ теорема 4 справедлива для класса $W_{\infty}^{Q}$.

Если $Q(D)=D^{r}$, то класс $W_{\infty}^{Q}$ совпадает с классом Соболева $W_{\infty}^{r}$, для которого точные значения изучаемых поперечников были найдены В. М. Тихомировым [9] . 
Сушествуют полиномы $Q$, нули которых находятся сколь угодно далеко от вещественной оси, а соответствуюшие им ядра $\Omega_{Q}$ являются ядрами, не повышающими осцилляции. Например, ядро $\Omega_{Q}$ для

$$
Q(D)=D\left(D^{2}+1^{2}\right) \cdots\left(D^{2}+m^{2}\right)
$$

является ядром, не повышающим осцилляции с $M=\{0, \pm 1, \cdots \pm m\}$ (см. [10]).

В общем случае полином $Q(D)$ может быть представлен в виде

$$
Q(D)=\prod_{j=1}^{k} Q_{j}(D)
$$

где $Q_{j}(D)$ - дифференциальные полиномы с вещественньпи коэффициентами такие, что $\operatorname{deg} Q_{j} \leqslant 2$. Из работы [3] следует, что $\Omega_{Q_{j}} \in \mathscr{K}\left(M_{j}, \delta_{j}\right)$, где

$$
M_{j}=\left\{m \in \mathbb{Z} \mid Q_{j}(i m)=0\right\}, \quad \delta_{j}=\pi / h\left(Q_{j}\right) .
$$

Поэтому, положив $\Phi x=\Omega_{Q_{k}} * \cdots * \Omega_{Q_{1}} * x$, получаем, что

$$
W_{\infty}^{Q}=\mathscr{F}_{\infty}(\Phi, M), \quad M=\bigcup_{j=1}^{k} M_{j} \quad\left(F=G=\mathrm{Id}, M_{0}=\varnothing\right) .
$$

Обозначим через $h_{\infty}^{\beta}\left(H_{\infty}^{\beta}\right)$ класс вещественных $2 \pi$-периодических функций, аналитически продолжаемых в полосу $S_{\beta}:=\{z \in \mathbb{C}|| \operatorname{Im} z \mid<\beta\}$ и удовлетворяющих в ней условию

$$
|\operatorname{Re} f(z)| \leqslant 1 \quad(|f(z)| \leqslant 1)
$$

Хорошо известно (см., например [11, с. 269]), что

$$
h_{\infty}^{\beta}=\left\{K_{\beta} * x \mid x \in B L_{\infty}\right\},
$$

где

$$
K_{\beta}(t)=1+2 \sum_{m=1}^{\infty} \frac{\cos m t}{\operatorname{ch} m \beta} .
$$

Ядро $K_{\beta}$ является $C V D$-ядром (см. [1, с. 62]). Таким образом,

$$
h_{\infty}^{\beta}=W_{\varnothing}\left(K_{\beta}\right) .
$$

Рассмотрим теперь класс $H_{\infty}^{\beta}$. В силу того, что функция $w=\frac{4}{\pi} \operatorname{arctg} z$ конформно отображает внутренность единичного круга на полосу $|\operatorname{Re} w|<1$, имеем

$$
f(\cdot) \in H_{\infty}^{\beta} \Longleftrightarrow \frac{4}{\pi} \operatorname{arctg} f(\cdot) \in h_{\infty}^{\beta} .
$$


Поэтому

$$
H_{\infty}^{\beta}=\left\{\varphi\left(K_{\beta} * x\right) \mid x \in B L_{\infty}\right\}
$$

где $\varphi(w)=\operatorname{tg} \frac{\pi}{4} w$. Поскольку $\operatorname{sign} \varphi(w)=\varphi(\operatorname{sign} w)$ при $w \in[-1,1]$, то нетрудно убедиться, что оператор

$$
F x=\varphi\left(K_{\beta} * x\right)
$$

принадлежит множеству $C V D(\varnothing, M, F)$ при всех $M$. Тем самым

$$
H_{\infty}^{\beta}=\mathscr{F}_{\infty}(\Phi, \varnothing), \quad \Phi x=F x \quad(k=0, G=F)
$$

Для дифференциального полинома $Q(D)$ с вещественньми коэффициентами через $h_{\infty}^{Q, \beta}\left(H_{\infty}^{Q, \beta}\right)$ обозначим класс вещественных $2 \pi$-периодических функций, аналитически продолжаемых в полосу $S_{\beta}$ и удовлетворяющих условию

$$
Q(D) f \in h_{\infty}^{\beta} \quad\left(Q(D) f \in H_{\infty}^{\beta}\right)
$$

В обозначениях (12) и (13) имеем

$$
h_{\infty}^{Q, \beta}=\mathscr{F}\left(\Phi_{1}, M\right), \quad H_{\infty}^{Q, \beta}=\mathscr{F}\left(\Phi_{2}, M\right)
$$

где $\Phi_{1} x=\Omega_{Q_{k}} * \cdots * \Omega_{Q_{1}} * K_{\beta} * x, \Phi_{2} x=\Omega_{Q_{k}} * \cdots * \Omega_{Q_{1}} * \varphi\left(K_{\beta} * x\right), M=\bigcup_{j=1}^{k} M_{j}$ (в первом случае $G=\mathrm{Id}$, а во втором $-G=F$ ).

Таким образом, из теоремы 4 вытекает

TEOPEMA 5. При всеx $n>2 h(Q)$

$$
\begin{aligned}
d_{2 n}\left(W, L_{\infty}\right) & =\lambda_{2 n}\left(W, L_{\infty}\right)=d^{2 n}\left(W, L_{\infty}\right)=i_{2 n}\left(W, L_{\infty}\right) \\
& =d_{2 n-1}\left(W, L_{\infty}\right)=\lambda_{2 n-1}\left(W, L_{\infty}\right)=d^{2 n-1}\left(W, L_{\infty}\right) \\
& =i_{2 n-1}\left(W, L_{\infty}\right)= \begin{cases}\left\|\Omega_{Q} * h_{n}\right\|_{\infty}, & W=W_{\infty}^{Q}, \\
\left\|\Omega_{Q} * K_{\beta} * h_{n}\right\|_{\infty}, & W=h_{\infty}^{Q, \beta}, \\
\left\|\Omega_{Q} * \varphi\left(K_{\beta} * h_{n}\right)\right\|_{\infty}, & W=H_{\infty}^{Q, \beta}\end{cases}
\end{aligned}
$$

При этом коэффициентьи Фурье $a_{0}(f), a_{1}(f), b_{1}(f), \ldots, a_{n-1}(f), b_{n-1}(f)$ являются оптимальными функиионалами для величин $i_{2 n-1}$ u $i_{2 n}$.

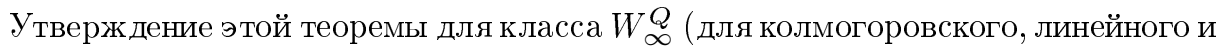
гельфандовского поперечников) было получено в работе [3]. Четные поперечники класса $H_{\infty}^{Q, \beta}$ при $Q(D)=D^{r}$ были найдены в работе [6].

Идея построения общей теории для гладкого и аналитического случаев неоднократно высказывалась профессором В. М. Тихомировым, которому автор признателен за полезные обсуждения. 


\section{Список литературы}

1. Pinkus A. n-Widths in Approximation Theory. Berlin: Springer-Verlag, 1985.

2. Нгуен Тхи Тхьеу Хоа. Наилучшие квадратурные формулы и методы восстановления функций, определяемых ядрами, не увеличивающими осцилляцию // Матем. сб. 1986. T. 130. № 1. C. 105-119.

3. Нгуен Тхи Тхвеу Хоа. Некоторые экстремальные задачи на классах функций, задаваемых линейньми дифференциальньми операторами // Матем. сб. 1989. Т. 180. № 10. C. $1355-1395$.

4. Осипенко K. Ю. Об $n$-поперечниках, оптимальных квадратурных формулах и оптимальном восстановлении функций, аналитических в полосе // Изв. РАН. Сер. матем. 1994. T. 58. № 4. C. 55-79.

5. Osipenko $K$. Yu. Exact values of $n$-widths and optimal quadratures on classes of bounded analytic and harmonic functions // J. Approx. Theory. 1995. V. 82. № 1. P. 156-175.

6. Osipenko K. Yu. Exact $n$-widths of Hardy-Sobolev classes // Constr. Approx. 1997. V. 13. P. $17-27$.

7. Karlin S. Total Positivity. V. I. Stanford: Stanford Univ. Press, 1968.

8. Крейн М.Г. К теории наилучшего приближения периодических функций // Докл. АН CCCP. 1938. T. 18. № 4-5. C. 245-251.

9. Тихомиров В. М. Наилучшие методы приближения и интерполирования дифференцируемых функций в пространстве $C[-1,1]$ // Матем. сб. 1969. Т. 80 (122). С. 290-304.

10. Нгуен Тхи Тхьеу Хоа. Оператор $D\left(D^{2}+1^{2}\right) \cdots\left(D^{2}+n^{2}\right)$ и тригонометрическая интерполяция // Anal. Math. 1989. Т. 15. №4. C. 291-306.

11. Ахиезер Н. И. Лекции по теории аппроксимации. М.: Наука, 1965.

МАТИ - Российский государственный технологический университет им. К.Э. Циолковского
Поступила в редакцию 13.05 .1996 